\title{
Costos médicos directos por intento de suicidio en pacientes del hospital mental de Antioquia, Colombia
}

\author{
Marisol Medina, ${ }^{1}$ Angie Vanessa Velásquez, ${ }^{1}$ Oscar Javier Ribero ${ }^{2}$ \\ y Natalia Trujillo ${ }^{1}$
}

Forma de citar

Medina M, Velásquez AV, Ribero OJ, Trujillo N. Costos médicos directos por intento de suicidio en pacientes del hospital mental de Antioquia, Colombia. Rev Panam Salud Publica. 2018;42:e129. https:/ / doi.org/10.26633/RPSP.2018.129

RESUMEN Objetivo. Analizar el comportamiento de los costos médicos directos en relación al número de intentos de suicidio y comparar los costos de atención cognitivo-conductual respecto al tratamiento convencional.

Métodos. Se cuantificaron los costos por prestación de servicios hospitalarios por intento de suicidio en 248 pacientes con diagnóstico de enfermedad mental atendidos en la empresa social del estado (E.S.E.) Hospital Mental de Antioquia y se implementó una evaluación de costo-consecuencia.

Resultados. Se encontró que los costos directos promedio de la atención de pacientes con cuatro o más intentos de suicidio fueron equivalentes a 5641 dólares estadounidenses (USD), con una diferencia de USD 5490 respecto al grupo con un solo intento. Además, dichos costos aumentaron conforme se incrementó el número de intentos. Por último, el diagnóstico de enfermedad mental ( $p$. ej., cronicidad), el método de intento y la necesidad de servicios especializados se relacionaron con el incremento en los costos directos.

Conclusiones. La implementación de estrategias preventivas desde la salud pública que evalúen y hagan seguimiento a los factores psicosociales podría reducir la presentación de la problemática y de sus costos médicos directos.

Palabras clave Salud mental; asignación de costos; intento de suicidio; trastornos mentales; factores de riesgo; Colombia.

Según la Organización Mundial de la Salud (OMS), para el 2015, el 29,1\% de los niños, adolescentes y adultos jóvenes del mundo presentaban algún tipo de trastorno mental (1). Estos grupos

\footnotetext{
1 Facultad Nacional de Salud Pública Universidad de Antioquia (UDEA), Medellín, Antioquia, Colombia. Enviar la correspondencia a Natalia Trujillo, natalia.trujillo@udea.edu.co

2 Servicio de Psiquiatría, Empresa Social del Estado (E.S.E) Hospital Mental de Antioquia, Colombia.
}

poblacionales, en especial los que manifiestan afectaciones del estado de ánimo y abuso de sustancias psicoactivas, tienen mayor riesgo de conductas autolesivas y suicidio consumado, lo que implica importantes costos sociales y económicos (2). Estos costos ascienden a miles de millones de dólares estadounidenses (USD) por año y suelen estimarse con base en el costo de atención del suicidio consumado. Esta estrategia de cuantificación, por una parte, invisibiliza el costo acumulado de los intentos previos del mismo paciente $y$, por otra, limita el direccionamiento de los recursos financieros hacia la intervención de factores asociados al intento, esto como una forma de prevenir su consumo futuro (3-7).

En el año 2015, el suicidio en la Región de las Américas representó 12,4\% de las causas externas de muerte, con tasas cercanas a $20 \%$ para Canadá, Cuba y

Este es un artículo de acceso abierto distribuido bajo los términos de la licencia Creative Commons Attribution-NonCommercial-NoDerivs 3.0 IGO, que permite su uso, distribución y reproducción en cualquier medio, siempre que el trabajo original se cite de la manera adecuada. No se permiten modificaciones a los artículos ni su uso comercial. Al reproducir un artículo no debe haber ningún indicio de que la OPS o el artículo avalan a una organización o un producto específico. El uso del logo de la OPS no está permitido. Esta leyenda debe conservarse, junto con la URL original del artículo. 
Estados Unidos de América (EE.UU.) (8). Los costos de esta problemática en EE.UU., por ejemplo, se estimaron en USD 25000 millones (9). En América Latina, Colombia es el tercer país con mayor número de suicidios consumados, con una tasa de 5,2 suicidios por cada 100000 habitantes en 2015 (10) y 10\% de incremento en 2016 (11). En ese año, la incidencia de intentos de suicidio osciló entre 10 a 40 veces por cada suicidio consumado (6) y $60 \%$ de estos reportaron historia de trastornos del estado de ánimo (12). Antioquia es uno de los departamentos de Colombia que mejor representa la problemática a nivel nacional (13).

En el país, el presupuesto invertido en salud mental es de 0,08\% del destinado a salud (14). Esto limita la oferta de servicios en esta área, lo que se refleja en recaídas, altas tasas de estancia hospitalaria asociadas a la reaparición sintomática y en la presentación de consecuencias letales o incapacitantes derivadas de la enfermedad mental (intentos de suicidios o suicidios consumados) $(9,15)$. En términos de carga de la enfermedad, el suicidio representó para Colombia 2,2 \% de los años potenciales de vida perdidos (APVP) para el 2002 (se carece de reportes actuales). Los altos APVP se debieron principalmente a la elevada prevalencia de muertes por suicidio en mujeres de 10-19 años y hombres de 20-29 años (16) y por la disminución de ingresos al sistema por pérdida de productividad laboral.

Los estudios sobre los costos derivados de la atención por intento de suicidio en la población con trastornos mentales son escasos; sin embargo, se estima la pertinencia de su análisis por el riesgo relativo que representa el intento de suicidio en la predicción de su consumación; la presencia de una relación incremental del $28,9 \%$ en el costo total anual de la atención de la enfermedad mental durante al año siguiente al intento de suicidio $(17,18)$; la relación del intento de suicidio y la enfermedad mental con los mayores costos sociales y sobre los sistemas financieros y de salud $(9,15$, $17,19,20)$ y la necesidad de establecer comparaciones costo-consecuencia entre intervenciones que han mostrado efectividad a nivel mundial frente a las ofertadas en países como Colombia, enfocadas en el manejo de la crisis aguda y durante el año siguiente al intento.

Por lo anterior, este estudio analizó el comportamiento de los costos de la atención en salud mental según el número de intentos de suicidio en pacientes con diagnóstico de enfermedad mental atendidos en la empresa social del estado (E.S.E.) Hospital Mental de Antioquia (HOMO) entre los años 2014 y 2016. Por otra parte, estableció la proyección de los costos de la atención cognitivo-conductual en comparación con la intervención convencional realizada en la institución. La evaluación económica de esta problemática resulta crucial para el direccionamiento, gestión y asignación de recursos en instituciones similares a la analizada en este estudio. Se espera, además, que la evidencia generada, complementada con estudios similares en la temática, sea útil para el desarrollo de políticas de salud mental enfocadas en la prevención y control de factores de riesgo; esto con el fin último de aportar en la determinación de focos de atención en salud mental en beneficio de la reducción de los costos sociales y económicos de esta problemática.

\section{MATERIALES Y MÉTODOS}

Se trata de un estudio analítico de costo-consecuencia, con fuente de información secundaria. Se incluyeron los costos médicos directos que comprenden la cantidad, tasa de uso y costo unitario de cada recurso facturado para el tratamiento y prestación de los servicios durante la atención del intento de suicidio (atención en servicios de urgencias, salario personal por fracción de tiempo que dedique en la atención del paciente, medicamentos, exámenes y ayudas diagnósticas usadas en este servicio), habitación, exámenes de laboratorio, fármacos prescritos y control médico). Se calcularon estos costos para cada paciente y se promediaron en función al número de intentos reportados en la historia clínica para el período en estudio, se conformaron cuatro grupos con uno, dos, tres y cuatro o más intentos, respectivamente. Los análisis se presentan, además, discriminados por las variables: hospitalización, estancia hospitalaria, tratamiento farmacológico, método de intento de suicidio (p. ej., corte en las muñecas, brazo o cuello), diagnóstico principal (p. ej., episodio depresivo) y número de intentos de suicidio.

La población objetivo fueron 248 pacientes con diagnóstico de enfermedad mental que ingresaron por intento de suicidio al Hospital Mental de Antioquia, Colombia entre los años 2014 y 2016.
De estos, 92 fueron hombres y 156 mujeres. La selección de la población se realizó con la aplicación de dos criterios: identificación de los reportes de individuos que ingresaron al servicio de urgencias por intento de suicidio, registrados con los códigos (X60-X84 y Z91) de la Clasificación Internacional de Enfermedades (CIE-10), y los reportados en el Sistema de Vigilancia Epidemiológico (SIVIGILA) por el mismo motivo. El uso de dos fuentes de información complementarias buscó prevenir el subregistro.

Se diseñó una base de datos en Microsoft Excel $2013^{\circledR}$ y en SPSS Statistics Base versión $22.0^{\circledR}$. Los datos recolectados se encuentran consignados en la historia clínica electrónica $\left(\mathrm{XEMCO}^{\circledR}\right)$ de la institución. A partir de esta, se evaluó la normalidad de la distribución de los costos con la prueba Kolgomorov-Smirnov y se calculó el valor de $P$ asociado al chi-cuadrado de Kruska1-Wallis para juzgar la significancia de la diferencia de costos derivados de la atención entre los grupos conformado respecto al número de intentos.

Por último, se empleó el criterio de extrapolación, según el cual se retomó un estudio previo de costo-consecuencia en el que se evaluó un tratamiento de corte cognitivo conductual que demostró utilidad en la disminución del riesgo de suicidio consumado en pacientes con trastornos mentales (p. ej., ansiedad, depresión mayor y trastornos adaptativos) (21). Este tratamiento se comparó con una intervención convencional enfocada en la atención aguda y durante el primer año del intento de suicidio, esto de acuerdo con las tarifas vigentes para Colombia en el año 2016. Para este fin, se contabilizó en el tratamiento cognitivo conductual: la intervención psicológica individual durante 45 minutos/ semana, una llamada de seguimiento por 15 minutos/semana -ambos durante un año- y $10 \%$ adicional de gastos de administración. Los costos se discriminaron en relación al número de intentos de suicidio y a la cantidad de pacientes que conformaban cada uno de los grupos de análisis. Este tratamiento se comparó con los gastos facturados para el tratamiento convencional empleado durante la atención del intento de suicidio (atención en servicios de urgencias, salario personal por fracción de tiempo que dedique en la atención del paciente, medicamentos, exámenes y ayudas diagnósticas usadas en este servicio), 
habitación, exámenes laboratorio, fármacos prescritos y control médico).

Todos los costos se estimaron en moneda local y luego se convirtieron a dólares corrientes al 31 de diciembre de 2016, según el tipo de cambio publicados por el Banco de la República de Colombia (1 dólar estadounidense [USD] = 3 000,71 pesos colombianos, $\mathrm{COP}$ ).

\section{Consideraciones éticas}

La investigación se adhirió a la Resolución 8430 de 1993 del Ministerio de Salud de Colombia, que la consideró sin riesgo, ya que se realizó con fuentes secundarias sin implementar intervenciones sobre los participantes (22). La investigación implementó análisis con datos agrupados por lo que no se revela información demográfica, socioeconómica o clínica individual de los participantes en el estudio. El comité de ética del Hospital Mental de Antioquia avaló y acompañó su desarrollo. La base de datos, según lo consignado en la Declaración de Helsinki (23), usó códigos alfanuméricos de identificación con el fin de mantener la privacidad de las historias clínicas. Se realizaron discusiones académicas con el objeto de evaluar los alcances de los resultados al interior de la institución, así como actividades de socialización de los resultados del estudio dirigidas a las directivas y cuerpo médico del hospital mental.

\section{RESULTADOS}

La población de estudio se caracterizó por tener un nivel de ingresos promedio de USD 105,25 (24) y ser, en su mayoría, de sexo femenino $(62,9 \%)$, con una edad entre 12 y 20 años, estar soltera y no tener hijos. Además, 53,23\% no ha terminado los estudios secundarios y $80,24 \%$ depende económicamente de terceros.

Los costos médicos totales de la atención por intento de suicidio entre 2014 y 2016 de 248 pacientes fueron de USD 270 321. Según el análisis de los datos, se evidencia una disminución del número de pacientes conforme aumenta el número de intentos de suicidio, lo que podría sesgar la estimación de los costos totales; sin embargo, se percibe un incremento en dichos costos, el cual se hace evidente al observar valores de USD 21994 para la atención de 151 pacientes con un intento frente a USD 136284 informados para 25 pacientes con cuatro o más intentos, con una diferencia de USD 114290 entre estos grupos (cuadro 1). El incremento de los costos directamente proporcional al aumento del número de intentos de suicidio se refleja para todos los grupos. Así, el costo promedio tiene un incremento de USD 858, 1806 y 2 641, respectivamente, en relación al gasto de la atención del intento de suicidio del grupo anterior (cuadro 1).

Se observa también una diferencia de los costos médicos directos promedio entre los pacientes que reciben servicios de hospitalización, tratamiento farmacológico o ambos respecto a los que no lo reciben equivalente a USD 3087 y 1 686, respectivamente (cuadro 1). Además, la estancia hospitalaria es de mayor costo conforme aumenta el número de días, la diferencia del costo promedio entre el período mínimo y el período máximo de atención de USD 12796 (cuadro 1).

Los costos médicos directos promedio más elevados en relación al mecanismo de intento son de USD 5 895, 3228 y 2 234, correspondientes a otras causas (p. ej., incineración), ahorcamiento y corte de cuello, respectivamente (cuadro 2). Los costos aumentan conforme se incrementa el número de intentos de suicidio; un ejemplo es la diferencia de los costos promedios de la atención de los pacientes que usaron como método el ahorcamiento, en el que se observa un incremento de USD 532, 842 y 10050 respecto al gasto de la atención del intento de suicidio del grupo anterior; se observa el mismo patrón para los demás métodos empleados en el intento (cuadro 2). Los mayores costos promedio asociados al diagnóstico principal de los pacientes con intento suicidio corresponden a retraso mental, otros (p. ej., distimia, insomnio no orgánico) episodio depresivo moderado y trastorno opositor desafiante, equivalentes a USD 3 065, 2 669, 2006 y 1370 , respectivamente (cuadro 3). Para los pacientes con un intento de suicidio,

CUADRO 1. Costos médicos directos totales y promedioa derivados de la atención por intento de suicidio en pacientes con diagnóstico de enfermedad mental, según número de intento, HOMO (2014-2016)

\begin{tabular}{|c|c|c|c|c|c|c|c|c|c|c|c|c|c|c|c|c|}
\hline \multirow{3}{*}{ Variable } & \multirow{3}{*}{ Categorías } & \multicolumn{15}{|c|}{ Número de intentos de suicidio } \\
\hline & & \multicolumn{3}{|c|}{ Uno } & \multicolumn{3}{|c|}{ Dos } & \multicolumn{3}{|c|}{ Tres } & \multicolumn{3}{|c|}{ Cuatro o más } & \multicolumn{3}{|c|}{ Total } \\
\hline & & $\mathrm{n}$ & $\Sigma$ & $\bar{x}$ & $n$ & $\Sigma$ & $\bar{x}$ & $n$ & $\Sigma$ & $\bar{x}$ & $\mathrm{n}$ & $\Sigma$ & $\bar{x}$ & $\mathrm{~N}$ & $\Sigma$ & $\bar{x}$ \\
\hline Hospitalización & Sí & 25 & 17410 & 696 & 19 & 45878 & 2415 & 13 & 59910 & 4608 & 16 & 115276 & 7205 & 73 & 238473 & 3267 \\
\hline \multirow{5}{*}{$\begin{array}{l}\text { Estancia hospitalaria } \\
\text { (días) }\end{array}$} & Ninguno & 123 & 3412 & 28 & 29 & 3363 & 116 & 10 & 3010 & 301 & 7 & 1897 & 271 & 169 & 11682 & 69 \\
\hline & $1-5$ & 3 & 209 & 70 & 3 & 2013 & 671 & 3 & 1498 & 499 & 3 & 3840 & 1280 & 12 & 7560 & 630 \\
\hline & $16-20$ & 2 & 2130 & 1065 & 2 & 1930 & 965 & 1 & 2050 & 2050 & 1 & 12426 & 12426 & 6 & 18536 & 3089 \\
\hline & $21-25$ & 2 & 1931 & 966 & 2 & 2737 & 1369 & 2 & 30734 & 15367 & 1 & 1664 & 1664 & 7 & 37066 & 5295 \\
\hline & $>$ & 1 & 1641 & 1641 & 1 & 24549 & 24549 & 0 & 0 & 0 & 3 & 38113 & 12704 & 5 & 64303 & 12861 \\
\hline \multirow{2}{*}{$\begin{array}{l}\text { Tratamiento } \\
\text { farmacológico }\end{array}$} & No & 114 & 1676 & 23 & 25 & 223 & 17 & 6 & 1042 & 347 & 6 & 1843 & 369 & 151 & 4784 & 50 \\
\hline & Sí & 37 & 20318 & 264 & 25 & 49999 & 1351 & 16 & 60779 & 3199 & 19 & 134441 & 6722 & 97 & 265537 & 1736 \\
\hline
\end{tabular}

$\mathrm{n}$, total pacientes para cada subgrupo (uno, dos, tres y cuatro o más intentos); $\Sigma$, sumatoria de los costos totales de atención de los pacientes por cada su subgrupo (uno, dos, tres y cuatro o más intentos); $\bar{X}$, costos promedios de atención en cada subgrupo (uno, dos, tres y cuatro o más intentos); N, total de pacientes atendidos.

a En dólares estadounidenses. 
CUADRO 2. Costos médicos directos totales y promedioa en relación al mecanismo del último intento de suicidio de pacientes con diagnóstico de enfermedad mental, según número de intentos, 2014-2016

\begin{tabular}{|c|c|c|c|c|c|c|c|c|c|c|c|c|c|c|c|}
\hline \multirow{3}{*}{ Método } & \multicolumn{15}{|c|}{ Número de intentos de suicidio } \\
\hline & \multicolumn{3}{|c|}{ Uno } & \multicolumn{3}{|c|}{ Dos } & \multicolumn{3}{|c|}{ Tres } & \multicolumn{3}{|c|}{ Cuatro o más } & \multicolumn{3}{|c|}{ Total } \\
\hline & $\mathrm{n}$ & $\Sigma$ & $\bar{x}$ & $\mathrm{n}$ & $\Sigma$ & $\bar{X}$ & $\mathrm{n}$ & $\Sigma$ & $\bar{x}$ & $\mathrm{n}$ & $\Sigma$ & $\bar{x}$ & N & $\Sigma$ & $\bar{x}$ \\
\hline Ahorcamiento & 6 & 808 & 135 & 7 & 4666 & 667 & 3 & 4528 & 1509 & 5 & 57796 & 11559 & 21 & 67798 & 3228 \\
\hline Corte en el brazo & 4 & 421 & 105 & 4 & 2576 & 644 & 2 & 715 & 357 & 2 & 11572 & 5786 & 12 & 15284 & 1274 \\
\hline Corte en el cuello & 4 & 4698 & 1174 & 1 & 11 & 11 & 0 & 0 & 0 & 2 & 10927 & 5463 & 7 & 15635 & 2234 \\
\hline Corte en la muñeca & 18 & 2837 & 158 & 10 & 4635 & 463 & 6 & 13342 & 2224 & 6 & 16659 & 2777 & 40 & 37473 & 937 \\
\hline Más de un método & 5 & 668 & 134 & 2 & 982 & 491 & 0 & 0 & 0 & 2 & 4588 & 2294 & 9 & 6238 & 693 \\
\hline Lanzarse al vacío & 5 & 1182 & 236 & 0 & 0 & 0 & 0 & 0 & 0 & 1 & 1664 & 1664 & 6 & 2845 & 474 \\
\hline Otro & 2 & 22 & 11 & 3 & 24601 & 8200 & 1 & 5084 & 5084 & 1 & 11558 & 11558 & 7 & 41264 & 5895 \\
\hline Total de costos & 151 & 21994 & 146 & 50 & 50222 & 1004 & 22 & 61821 & 2810 & 25 & 136284 & 5451 & 248 & 270321 & 1090 \\
\hline
\end{tabular}

CUADRO 3. Costos totales y promedioa del intento de suicidio, según diagnóstico principal y número de intentos, 2014-2016

\begin{tabular}{|c|c|c|c|c|c|c|c|c|c|c|c|c|c|c|c|}
\hline \multirow{3}{*}{ Método } & \multicolumn{15}{|c|}{ Número de intentos de suicidio } \\
\hline & \multicolumn{3}{|c|}{ Uno } & \multicolumn{3}{|c|}{ Dos } & \multicolumn{3}{|c|}{ Tres } & \multicolumn{3}{|c|}{ Cuatro o más } & \multicolumn{3}{|c|}{ Total } \\
\hline & $\mathrm{n}$ & $\Sigma$ & $\bar{x}$ & $\mathrm{n}$ & $\Sigma$ & $\bar{x}$ & $n$ & $\Sigma$ & $\bar{x}$ & $\mathrm{n}$ & $\Sigma$ & $\bar{X}$ & N & $\Sigma$ & $\bar{x}$ \\
\hline Episodio depresivo grave & 7 & 854 & 122 & 4 & 83 & 21 & 2 & 2400 & 1200 & 4 & 12317 & 3079 & 17 & 15653 & 921 \\
\hline Episodio depresivo leve & 10 & 1019 & 102 & 2 & 8025 & 4012 & 1 & 17 & 17 & 0 & 0 & 0 & 13 & 9061 & 697 \\
\hline Episodio depresivo moderado & 34 & 6251 & 184 & 9 & 27841 & 3093 & 5 & 34458 & 6892 & 6 & 39791 & 6632 & 54 & 108341 & 2006 \\
\hline Trastorno depresivo recurrente & 10 & 1928 & 193 & 4 & 2862 & 715 & 2 & 1034 & 517 & 0 & 0 & 0 & 16 & 5824 & 364 \\
\hline Trastorno de estrés agudo & 7 & 2756 & 394 & 3 & 61 & 20 & 0 & 0 & 0 & 0 & 0 & 0 & 10 & 2817 & 282 \\
\hline $\begin{array}{l}\text { Trastorno de personalidad } \\
\text { emocionalmente inestable }\end{array}$ & 15 & 652 & 43 & 5 & 640 & 128 & 2 & 559 & 280 & 4 & 7752 & 1938 & 26 & 9604 & 369 \\
\hline Trastorno opositor desafiante & 1 & 9 & 9 & 1 & 2730 & 2730 & 0 & 0 & 0 & 0 & 0 & 0 & 2 & 2739 & 1370 \\
\hline $\begin{array}{l}\text { Trastornos mentales y del } \\
\text { comportamiento por psicoactivos }\end{array}$ & 13 & 219 & 17 & 2 & 858 & 429 & 4 & 11655 & 2914 & 0 & 0 & 0 & 19 & 12732 & 670 \\
\hline Total de costos & 151 & 21994 & 146 & 50 & 50222 & 1004 & 22 & 61821 & 2810 & 25 & 136284 & 5451 & 248 & 270321 & 1090 \\
\hline
\end{tabular}

$\mathrm{n}$, total de pacientes para cada subgrupo (uno, dos, tres y cuatro o más intentos); $\Sigma$, sumatoria de los costos totales de atención de los pacientes por cada su subgrupo (uno, dos, tres y cuatro o más intentos); $\bar{X}$, costos promedios de atención en cada subgrupo (uno, dos, tres y cuatro o más intentos); N, total de pacientes atendidos.

a En dólares estadounidenses.

los diagnósticos de mayores costos promedio son trastorno de estrés agudo y otro; mientras que, para dos intentos, son el episodio depresivo leve, moderado y el trastorno opositor desafiante equivalentes a USD 4 012, 3093 y 2 730, respectivamente.

De los pacientes identificados con tres intentos de suicidio, el episodio depresivo moderado, el trastorno de adaptación y los trastornos mentales del comportamiento debido al uso de sustancias psicoactivas describen costos promedio iguales a USD 6 892, 3331 y 2 914, respectivamente; mientras que los mayores costos se describen en el grupo con cuatro o más intentos para otros, retraso mental y trastorno afectivo bipolar equivalentes a USD 13 181, 11558 y 7 752, respectivamente (cuadro 3). De igual forma, los costos discriminados por diagnóstico se incrementan de manera general en relación al número de intentos.

Se observa que los costos por intento de suicidio en los pacientes con enfermedad mental no tienen una distribución normal (cuadro 4). Posteriormente, con base en este resultado se implementó la prueba Kruskal-Wallis $\left(\chi^{2}=60,147 ; P=0,000\right)$ que indica 
CUADRO 4. Evaluación de la distribución de la normalidad de la distribución de los costos médicos directos entre los grupos de número de intentos

\begin{tabular}{|c|c|c|c|c|c|}
\hline & \multirow{2}{*}{$\mathrm{N}^{0}$ de intentos } & \multirow{2}{*}{$\mathrm{n}$} & \multirow{2}{*}{ Rango promedio } & \multicolumn{2}{|c|}{ Kolgomorov-Smirnov } \\
\hline & & & & Estadístico & Valor de $P$ \\
\hline \multirow[t]{3}{*}{ Costos } & 1 & 151 & 100,56 & 0,399 & 0,000 \\
\hline & 2 & 50 & 133,01 & 0,392 & 0,000 \\
\hline & $\geq 4$ & 25 & 199,12 & 0,28 & 0,000 \\
\hline
\end{tabular}

CUADRO 5. Comparación de $\operatorname{costos}^{\mathrm{a}}$ por atención cognitivo-conductual respecto a la convencional por intento de suicidio en pacientes con enfermedad mental

\begin{tabular}{|c|c|c|c|c|c|c|c|c|c|c|c|c|c|c|c|}
\hline \multirow{3}{*}{ Atención } & \multicolumn{15}{|c|}{ Número de intentos de suicidio } \\
\hline & \multicolumn{3}{|c|}{ Uno } & \multicolumn{3}{|c|}{ Dos } & \multicolumn{3}{|c|}{ Tres } & \multicolumn{3}{|c|}{ Cuatro o más } & \multicolumn{3}{|c|}{ Total } \\
\hline & $\mathrm{n}$ & $\Sigma$ & $\bar{x}$ & $\mathrm{n}$ & $\Sigma$ & $\bar{x}$ & $n$ & $\Sigma$ & $\bar{x}$ & $n$ & $\Sigma$ & $\bar{x}$ & $\mathrm{~N}$ & $\Sigma$ & $\bar{x}$ \\
\hline Cognitivo-conductual & 151 & 23470 & 155 & 50 & 7772 & 155 & 22 & 3420 & 155 & 25 & 3886 & 155 & 248 & 38548 & 155 \\
\hline Convencional & 151 & 21994 & 146 & 50 & 50222 & 1004 & 22 & 61821 & 2810 & 25 & 136284 & 5451 & 248 & 270321 & 1090 \\
\hline Costo-consecuencia & 151 & -1476 & -10 & 50 & 42450 & 849 & 22 & 58402 & 2655 & 25 & 132398 & 5296 & 248 & 231773 & 935 \\
\hline
\end{tabular}

diferencias significativas de los costos entre los grupos categorizados según el número de intentos de suicidio.

Por último, se observan mayores costos para el tratamiento cognitivo-conductual respecto al convencional estimado para Colombia para el grupo de un intento. Sin embargo, al reconocer que el primero limita la recidiva, se puede sugerir que su uso durante los dos años siguientes al primer intento podría implicar un ahorro equivalente a USD 248 327. Se observa, además, un ahorro entre USD 42450 y 132389 por recibir tratamiento cognitivo-conductual en comparación con la atención convencional en pacientes reincidentes (cuadro 5). En general, significaría para el sistema de salud un ahorro total equivalente a USD 231773 (cuadro 5). De realizar la intervención durante los tres años de estudio en los 248 pacientes, el ahorro sería de USD 154678.

\section{DISCUSIÓN}

Este estudio tuvo como objetivo analizar el comportamiento de los costos de la atención en salud, según el número de intentos de suicidio en pacientes con diagnóstico de enfermedad mental, atendidos en la E.S.E. Hospital Mental de Antioquia entre el 2014 y 2016, así como, proyectar los costos de una intervención cognitivo-conductual que demostró mayor efectividad en la prevención del suicidio consumado con respecto al tratamiento convencional usado en Colombia. Los resultados obtenidos indican que los costos médicos directos promedio de la atención en salud incrementan de manera significativa en relación con el número de intentos de suicidio (cuadro 1); que los intentos con mecanismos de mayor letalidad como ahorcamiento, corte en cuello $\mathrm{u}$ otro ( $\mathrm{p}$. ej., incineración) significan los mayores costos promedio en la atención en salud (cuadro 2); que aun cuando el trastorno depresivo recurrente incrementa el riesgo de reincidencia por intento de suicidio, este significa uno de los menores costos promedios (USD 364) en la atención en salud mental, solo por encima de la esquizofrenia y el trastorno de estrés agudo (cuadro 3) y que el tratamiento cognitivo-conductual, en comparación con el convencional, puede significar para el sistema de salud una reducción en los costos médicos directos por intento de suicido (cuadro 5).

Los costos médicos directos en salud tras un intento de suicidio son significativos y aumentan conforme se presentan nuevos intentos. Un estudio sobre el costo de atención hospitalaria por suicidio atribuye principalmente el incremento en los costos a la atención psiquiátrica, al manejo de las secuelas físicas del intento $\mathrm{y} / \mathrm{o}$ al inminente riesgo del consumo del suicidio (25), lo cual es congruente con el aumento en la demanda de servicios es- pecializados al incrementar el número de intentos. De manera complementaria, un estudio realizado en Irlanda ha descrito costos similares a los encontrados en esta investigación. En este, se identificó un incremento de los costos de atención medica del paciente que casi se duplica durante el primer año tras un intento, ya que pasa de 367264 euros a 715019 euros (costos totales de 539 personas de los tres países) (25). Este incremento en los costos se atribuye a la presentación de uno o más intentos nuevos durante el período de observación, así como a la utilización de métodos de mayor letalidad y al diagnóstico de trastorno de adaptación y retraso mental (26). Esto es similar a lo observado en el presente estudio, en donde este último representó los mayores costos promedio en comparación con los demás diagnósticos.

Por su parte, el aumento de los costos en función de la presentación de nuevos intentos puede responder a la necesidad de desplegar protocolos más conservadores de atención psiquiátrica, por cuanto el riesgo de consumar el suicidio tras cada nuevo intento se ve aumentado en cinco a seis veces (19). Así, intentos que usen como método el ahorcamiento, por ejemplo, son responsables de cerca del $50 \%$ de muertes por suicidio en la mayoría de los países, razón por la cual se requieren protocolos de atención especializada y estancias hospitalarias más prolongadas $(19,27)$ con un consecuente incremento de los 
costos directos. Por otra parte, los pacientes denominados "suicidas crónicos" aun cuando tienden a usar métodos de menor letalidad, también requieren protocolos conservadores, porque se autoinfringen lesiones que derivan en secuelas permanentes para la salud, las cuales reduce su capacidad de recuperación completa y facilitan el eventual consumo del suicidio (25, 26, 28-30).

De manera complementaria, se ha informado que los trastornos del ánimo, el abuso de sustancias psicoativas y las conductas antisociales se asocian con mayor frecuencia con el intento de suicidio $(31,32)$. En particular, los trastornos depresivos incrementan diez veces la probabilidad de consumar el suicidio $y, a$ su vez, se relacionan con la reincidencia de intentos en adolescentes, lo que deriva en intentos más letales (50\% de los casos recurrieron a métodos más violentos) o mayor mortalidad (28, 33-35). Es relevante el alto riesgo que significa el trastorno depresivo mayor (TDM) $(\mathrm{OR}=3,2)$, por cuanto el suicidio y su tentativa puede estar presente (alrededor del 50\% de pacientes con TDM mueren dentro de los 12 meses siguientes a un intento) $(26,28$, $32,34)$. Por lo anterior, la escasa inversión en la atención en presencia de TDM se traduce en un riesgo nueve veces mayor de recidiva que los demás diagnósticos en la población observada. Este riesgo podría disminuir en presencia de tratamientos de corte cognitivo-conductual, que además, parecen representar una alternativa viable en la reducción de pérdida de vidas y de los costos derivados de la atención de los pacientes de la institución de estudio.

La atención primaria es el primer recurso al que acceden la mayoría de personas con problemas de salud mental: sin embargo, el nivel de direccionamiento desde estos hacia servicios psicosociales especializados es insuficiente (36). En este sentido, se describen limitaciones en el acceso a opciones terapéuticas continuas, como las de corte cognitivoconductual, descritas como una opción efectiva, plausible y de menor costo a mediano/largo plazo para disminuir los síntomas y discapacidad derivada del trastorno; esto en comparación con modelos de atención enfocados principalmente en la resolución de la fase aguda asociada al evento (36). De acuerdo con los resultados de esta investigación, la implementación de modelos de corte cognitivo-conductual parecen ser una estrategia de mayor eficiencia económica al compararla con los convencionales (cuadro 5) (36).

Si bien, la presente investigación no explora de manera directa la costo-efectividad de intervenciones diferentes a la de corte cognitivo-conductual, se sugiere que, en concordancia con otros estudios, los beneficios de monoterapias de tipo farmacológico o de intervenciones en crisis pueden ser menos claros en comparación con la implementación de estrategias de promoción y prevención en niveles primarios de salud $(15,20)$. Esto, además, puede significar menor pérdida de la productividad por enfermedad mental, disminución en la demanda de servicios especializados, disminución en la tasa de discapacidad por intento y, con ello, una posible disminución en la pérdida de vidas de personas jóvenes $(17,36)$.

\section{CONCLUSIONES}

El aumento en los costos médicos directos para la institución analizada en esta investigación es consistente con los factores de riesgo epidemiológico con frecuencia asociados con la consumación del suicidio descrito en estudios previos. En este sentido, implementar estrategias enfocadas en acciones preventivas accesibles y sostenibles como el tratamiento cognitivo-conductual en niveles primarios de atención que, además, realicen intervención de los factores priorizados como los analizados en este estudio (diagnóstico principal, mayor número de intentos de suicidio, método de intento de mayor letalidad y altas estancias hospitalarias), podría aportar en la reducción del riesgos de pérdidas de vida en la población observada y, por ende, en los costos directos de su intervención.

En cuanto a las limitaciones de esta investigación, su alcance se restringe a la consideración del HOMO como centro de referencia del departamento de Antioquia, Colombia, por lo que las conclusiones no deben entenderse como un reflejo de la dinámica de la problemática a nivel nacional o internacional. En este sentido, no es posible generar conclusiones directas sobre las causas necesarias para reducir los riesgos de vida y el aumento de los costos derivados por internación. Los costos estimados no incluyen los relacionados con atención en urgencias, hospitalización y unidad de cuidados intensivo de otras instituciones, desde donde fueron derivados los pacientes al HOMO.

Agradecimientos Los autores agradecen a la E.S.E Hospital Mental de Antioquia-Centro de Investigación por facilitar los elementos posibles para culminar con éxito este proyecto, en especial a Isabel Pérez Villegas que nos acompañó durante todo el proceso y, de igual forma, a nuestro maestro Emmanuel Nieto López.

Conflicto de intereses Ninguno declarado por los autores.

Declaración Las opiniones expresadas en este manuscrito son responsabilidad del autor y no reflejan necesariamente los criterios ni la política de la RPSP/ PAJPH y/o de la OPS.

\section{REFERENCIAS}

1. Organización Mundial de la Salud (OMS). 10 datos sobre la salud mental. Disponible en: http:/ / www.who.int/features / factfiles / mental_health/mental_health_facts/es/ index4.html Acceso el 14 de abril de 2016.

2. Macana Tuta NL. Comportamiento del suicidio, 2011. Disponible en: http:// www.medicinalegal.gov.co/documents/ 10180 / 34616/6F-11-Suicidio.pdf / 6b2966e7-cbcb-4618-a3c3-af5cd111629e Acceso el 14 de abril de 2016.

3. Gómez-Restrepo C, Rodríguez Malagón N, De Romero LC, Pinilla GC, López LE. Suicidio y lesiones autoinfligidas, Colombia, 1973-1996. Rev Colomb Psiquiatr. 2002;31(2):123-36. Disponible en: http://www.scielo.org.co/scielo.php? script $=$ sci_arttext\&pid=S0034-745020020 00200003\&lng $=$ en $\& n r m=$ iso $\&$ tlng $=$ es Acceso el 14 de abril de 2016.

4. Organización Mundial de la Salud. Suicidio. 2017. Disponible en: http:/ /www.who.int/ mediacentre/factsheets/fs398/es/

5. Organización Mundial de la Salud. Salud mental: nuevos conocimientos, nuevas esperanzas. Ginebra: OMS; 2001. Disponible en: http://www.who.int/whr/2001/en/ 
whr01_djmessage_es.pdf?ua=1 Acceso el 14 de abril de 2016.

6. Guilbert Reyes W. Epidemiología de la conducta suicida. Rev Cuba Med Gen Integr. 2009;18(2):139-42. Disponible en: http:// scielo.sld.cu/scielo.php?script=sci_arttext\&pid=S086421252002000200007\&lng= es\&nrm=iso\&tlng=es

7. Instituto de Medicina Legal y Ciencias Forenses. Herramienta para la interpretación, intervención y prevención de lesiones de causa externa en Colombia; 2014. Disponible en: http://www.medicinalegal.gov.co/documents / 10180/188820/ FORENSIS + 2013+3-+suicidio.pdf / 65a683b4-38b2-46a4-b32af2a0884b25bf

8. Hérnandez Soto PA, Villareal Casate RE. Algunos datos entorno a la conducta suicida. MEDISAN [Internet]. 2015;19(8): 1051-8. Disponible en: http://scielo.sld. $\mathrm{cu} / \mathrm{pdf} / \mathrm{san} / \mathrm{v} 19 \mathrm{n} 8 / \mathrm{san} 14198$.pdf. Acceso el 14 de abril de 2016

9. Latimer EA, Garièpy G, Greenfield B. Cost-effectiveness of a rapid response team intervention for suicidal youth presenting at an emergency department. Can J Psychiatry. 2014;59(6):310-8. Disponible en: https:// www.ncbi.nlm.nih.gov/pubmed/25007405 Acceso el 14 de enero de 2018.

10. Instituto de Medicina Legal y Ciencias Forenses. Comportamiento del suicidio. Colombia, 2015. Violencia autoinfligida, desde un enfoque forense. Medellín; 2015. Disponible en: http:/ / www.medicinalegal. gov.co/documents / 88730/3418907/7. +SUICIDIOS.pdf/ 01c41af2-27cf-4932ae14-234d1eeaf425

11. Instituto Nacional de Medicina Legal y Ciencias Forenses. Grupo Centro de Referencia Nacional sobre Violencia. Forensis 2016, Datos para la vida. Colombia; 2016. Disponible en: http://www.medicinalegal.gov.co/documents/20143/49526/ Forensis+2016.+Datos+para+la+vida.pdf

12. Arango Dávila CA, Carlos J, Fernández R, Moreno M. Análisis de los aspectos asociados a la enfermedad mental en Colombia y la formación en psiquiatría. Rev Colomb Psiquiat. 2008;37(4):538-63.

13. Alcaldía de Medellín. Plan de Salud Municipal. Medellín; 2012. Disponible en: https://www.medellin.gov.co/irj/go/ $\mathrm{km} / \mathrm{docs} / \mathrm{pccdesign} / \mathrm{Sub}$ ortaldel Ciudadano 2/PlandeDesarrollo_0 15/ InformacinGeneral/SharedContent/ Documentos/instrumentos/ps/PLAN DE SALUD MUNICIPAL.pdf

14. Martínez Hernández MA, Bataille Savón A, Duccase Pilón M, Borlot Vargas N. Costo hospitalario de pacientes diagnosticados con retraso mental. Rev Cuba Med Milit. 2002;31(3):188-93. Disponible en: http:// scielo.sld.cu/pdf/mil/v31n3/mil06302.pdf

15. Medina Pérez OA, Díaz Téllez AS, Rozo David AJ. Caracterización del suicidio en adolescentes de Antioquia, Colombia, 2000-2010. Rev Fac Med. 2015;63(3):431-8. Disponible en: http:/ /www.scielo.org.co/ pdf/rfmun/v63n3/v63n3a10.pdf Acceso el 14 de abril de 2016.

16. Cendales R, Vanegas C, Fierro M, Córdoba $\mathrm{R}$, Olarte A. Tendencias del suicidio en Colombia, 1985-2002. Rev Panam Salud Publica. 2007;22(4):231-8. Disponible en: http:/ / www.scielosp.org/scielo.php? script=sci_arttext\&pid=S1020-49892007000 900002\&lng=en\&nrm=iso\&tlng=es Acceso el 16 de abril de 2016.

17. Wagner FA, González Forteza C, Sánchez García S, García Peña C, Gallo JJ. Enfocando la depresión como problema de salud pública en México. Salud Mental. 2012;35(1): 3-11. Disponible en: http://www.scielo. org. $\mathrm{mx} / \mathrm{pdf} / \mathrm{sm} / \mathrm{v} 35 \mathrm{n} 1 / \mathrm{v} 35 \mathrm{n} 1 \mathrm{a} 2 . \mathrm{pdf}$ Acceso el 14 de abril de 2016

18. Góngora Salgado AC. Factores asociados a intento suicida en pacientes con antecedente psicótico. Universidad CES, Universidad del Rosario; 2011. Disponible en: http:// repository.urosario.edu.co/bitstream/ handle/10336/2672/40325223-2011.pdf? sequence=1 Acceso el 14 de abril de 2016.

19. García J, Palacio C, Arias S, Ocampo M, Calle J, Restrepo D, et al. Características asociadas al riesgo de suicidio valorado clínicamente en personas con intento reciente. Rev Colomb Psiquiat. 2007;36(4):610-27. Disponible en: http://www.scielo.org.co/ pdf/rcp/v36n4/v36n4a03.pdf

20. Gómez Restrepo C, Malagón Rodríguez N, Bohórquez A, Diazgranados N, García Ospina MB, Fernández C. Factores asociados al intento de suicidio en la población colombiana. Rev Colomb Psiquiatr. 2002; 26(4):271-86. Disponible en: http://www. scielo.org.co/pdf/rcp/v31n4/v31n4a02.pdf

21. Gobierno de Navarra, España. Prevención y actuación ante conductas suicidas. Disponible en: http://www.navarra.es/ NR/ rdonlyres / 1C0C8294-D0FD-405FB7CC-85CAFFBDC9BB/291404/00Protoc oloPrevencionSuicidio3.pdf Acceso el 14 de febrero de 2018.

22. Ministerio de Salud de Colombia. Res. No 8430 de 1993. Bogotá D.C.; 1993. Disponible en: https://www.minsalud.gov.co/sites/ rid/Lists/BibliotecaDigital/RIDE/DE/ DIJ/RESOLUCION-8430-DE-1993.PDF Acceso el 14 de abril de 2017.

23. Manzini JL. Declaración de Helsinki: principios éticos para la investigación médica sobre sujetos humanos. Acta Bioethica. 2000;(2). Disponible en: https://scielo. conicyt.cl/pdf/abioeth/v6n2/art10.pdf

24. Departamento Administrativo Nacional de Estadísticas. Ingresos y gastos de los hogares 2006-2007. Disponible en: https:// www.dane.gov.co/index.php/estadisticas-por-tema / pobreza-y-condiciones-devida/ingresos-y-gastos-de-los-hogares Acceso el 14 de abril de 2016.

25. O’Sullivan $M$, Laulor $M$, Corcoran $P$, Kelleher MJ. The cost of hospital care in the year before and after parasuicide. Crisis. 1999;20(4):178-83. Disponible en: https:// www.ncbi.nlm.nih.gov/pubmed/10680285 Acceso el 14 de abril de 2016.

26. Resa García E, Braquehais Conesa D. El paciente suicida. En: Elseiver España SL. Manual de urgencias psiquiátricas. 2ed. 2009:165-96. Disponible en: https: / /aplicacionesbiblioteca.udea.edu.co:2077/\#!/content/book/3-s2.0-B9788445820247500058 Acceso el 14 de abril de 2016.

27. Baader MT, Urra PE, Millán AR, Yéñez ML. Algunas consideraciones sobre el intento de suicidio y su enfrentamiento. Rev Med Clin Condes. 2011;22(3):303-9. Disponible en: http:/ /www.clc.cl/clcprod/media/contenidos/pdf/MED_22_3/303309-dr-baader.pdf

28. Subgerencia cultural del Banco de la República de Colombia. Capítulo 1. De los derechos fundamentales. En: Constitución Política de Colombia. Bogotá, D.C.; 2000. Disponible en: http:/ / www.banrepcultural. org/blaavirtual/derecho/constitucionpolitica-de-colombia-1991/titulo-2capitulo-1

29. Lozano Serrano C, Huertas Patón A Martínez Pastor CJ, Terrazas Ezquiaga E, García Camba de la Muela E, Rodríguez Salvanés FJ. Descriptive analysis of parasuicide in the psychiatric emergencies. Rev Asoc Esp Neuropsiq. 2004;91:11-22. Disponible en: http://scielo.isciii.es/scielo. php ?script $=$ sci_arttext $\&$ pid $=S 0211$ 57352004000300002

30. Clavijo Quiceno J, Serna Sernaitis M. Propuesta de intervención para el fortalecimiento de habilidades sociales y emocionales en adolescentes con ideación suicida de la ciudad de Medellín. Medellín Universidad del CES; 2010. Disponible en: http:/ / bdigital.ces.edu.co:8080/ repositorio/bitstream/10946/1200/2/FINAL TESIS_MANUELA_JULIANA.pdf

31. Morales González A, Jaramillo López C. Factores asociados a letalidad de intentos de suicidio en sujetos con trastorno depresivo mayor. Rev Colomb Psiquiatr. 2009;38(3):446-63. Disponible en: http:// www.scielo.org.co/pdf/rcp/v38n3/ v38n3a05.pdf Acceso el 9 de agosto de 2016.

32. Pacheco B, Peralta López P. La conducta suicida en la adolescencia y sus condiciones de riesgo. Ars Medica. 2015;40(1):47-55. Disponible en: http://www.arsmedica.cl/ index.php / MED/article/view /38/13 Acceso el 9 de agosto de 2016.

33. Sanchez Aguilar ML. Trastorno depresivo y suicidio. Universidad de Salalmanca; 2014. Disponible en: https:/ /gredos.usal.es/jspui/ bitstream/10366/127378/1/DPPMMLHM AguilarSanchez_TrastornodepresivoL.pdf Acceso el 14 de abril de 2016.

34. Hernández Soto PA, Villareal Casate RE. Algunas especificidades en torno a la conducta suicida. MEDISAN. 2015;19(8): 1051-8. Disponible en: http://scielo.sld. cu/pdf/san/v19n8/san14198.pdf Acceso el 14 de abril de 2016.

35. Fava G, Ruini C, Sonino N. Tratamiento de la depresión recurrente. Enfoque Psicoterapéutico y Psicofarmacológico Secuencial. SIIC. 2003;17(15):1109-17. Disponible en: http://www.bago.com/bagoarg/biblio/psiqweb213.htm Acceso el 14 de abril de 2016.

36. Arias López HA. Factores de éxito en programas de prevención del suicidio. Revista Vanguardia Psicológica. 2013(2):215-25. Disponible en: https://dialnet.unirioja. es/descarga/articulo/4815157.pdf Acceso el 14 de enero de 2018.

Manuscrito recibido el 27 de febrero de 2018 Aceptado para su publicación, tras revisión, el 22 de mayo de 2018. 
ABSTRACT

Direct medical costs of suicide attempts by patients in Columbia's Antioquia Mental Hospital

Objective. Analyze the behavior of direct medical costs in relation to the number of suicide attempts and compare the costs of cognitive-behavioral therapy with those of conventional treatment.

Methods. The cost of hospital services for attempted suicide was quantified for 248 patients with a diagnosis of mental illness treated at the Antioquia Mental Hospital, a state social enterprise (E.S.E.), and a cost-outcome analysis was performed.

Results. It was found that the average direct cost of care for patients with four or more suicide attempts was equivalent to US\$ 5,641, a US\$ 5,490 difference vis-à-vis the group with a single attempt. Moreover, the cost increased with the number of attempts. Finally, the diagnosis of mental illness (e.g., chronicity), the method used in the suicide attempt, and the need for specialized services were associated with the increase in direct costs.

Conclusions. The use of a public health approach involving preventive strategies that assess and monitor psychosocial factors could reduce the problem and its direct medical costs.

Keywords Mental health; cost allocation; suicide, attempted; mental disorders; risk factors; Colombia.

RESUMO

\section{Custos médicos diretos por tentativa de suicídio em pacientes do hospital de saúde mental de Antioquia, Colômbia}

Palavras-chave
Objetivo. Examinar o comportamento dos custos médicos diretos em relação ao número de tentativas de suicídio e comparar o custo da terapia cognitivocomportamental e do tratamento convencional.

Métodos. O custo por prestação de serviços hospitalares por tentativa de suicídio foi mensurado em 248 pacientes com diagnóstico de doença mental atendidos no Hospital Mental de Antioquia, um serviço da previdência social do Estado, e foi conduzida uma análise de custo-consequência.

Resultados. Verificou-se que os custos diretos médios do atendimento de pacientes com quatro ou mais tentativas de suicídio foram equivalentes a US\$ 5.641, com uma diferença de US\$ 5.490 em relação aos custos para os pacientes com uma única tentativa de suicídio. Estes custos aumentaram conforme aumentou o número de tentativas. O diagnóstico de doença mental (por exemplo, doença crônica), o método de tentativa de suicídio e a necessidade de serviços especializados foram relacionados ao aumento dos custos diretos.

Conclusões. A implementação de estratégias de prevenção de uma perspectiva de saúde pública para avaliar e monitorar os fatores psicossociais poderia contribuir para reduzir a ocorrência do problema e os custos médicos diretos correspondentes.

Saúde mental; alocação de custos; tentativa de suicídio; transtornos mentais; factores de risco; Colômbia. 